\title{
Prolongation Structures and $N$-Soliton Solutions for a New Nonlinear Schrödinger-Type Equation via Riemann-Hilbert Approach
}

\author{
Yuxin Lin, Yong Fang $(\mathbb{D}$, and Huanhe Dong $\mathbb{D}$ \\ College of Mathematics and System Sciences, Shandong University of Science and Technology, Qingdao 266590, Shandong, China \\ Correspondence should be addressed to Yong Fang; fangyong@sdust.edu.cn and Huanhe Dong; mathsdong@126.com
}

Received 9 May 2019; Accepted 24 June 2019; Published 11 July 2019

Academic Editor: Luigi Rodino

Copyright (C) 2019 Yuxin Lin et al. This is an open access article distributed under the Creative Commons Attribution License, which permits unrestricted use, distribution, and reproduction in any medium, provided the original work is properly cited.

In this paper, a new integrable nonlinear Schrödinger-type (NLST) equation is investigated by prolongation structures theory and Riemann-Hilbert (R-H) approach. Via prolongation structures theory, the Lax pair of the NLST equation, a $2 \times 2$ matrix spectral problem, is derived. Depending on the analysis of red the spectral problem, a R-H problem of the NLST equation is formulated. Furthermore, through a specific R-H problem with the vanishing scattering coefficient, $N$-soliton solutions of the NLST equation are expressed explicitly. Moreover, a few key differences are presented, which exist in the implementation of the inverse scattering transform for NLST equation and cubic nonlinear Schrödinger (NLS) equation. Finally, the dynamic behaviors of soliton solutions are shown by selecting appropriate spectral parameter $\lambda$, respectively.

\section{Introduction}

In recent decades, nonlinear partial differential equations (PDEs) play a significant role in mathematics and theoretical physics, which have attracted much attentions in soliton theory and integrable system $[1,2]$. Since exact solutions $[3,4]$ of the integrable equations $[5,6]$ can describe and explain many natural phenomena [7], the study of integrable equations has become a hot topic. It is well known that inverse scattering transform $[8,9]$ is a very important theory for solving exact solutions of integrable equations. There are two typical techniques for gaining inverse scattering: one is using Gel' fand-Levitan-Marchenko equations and the other is formatting a Riemann-Hilbert $(\mathrm{R}-\mathrm{H})$ problem. The former has a complicated calculation procedure, while the latter can provide an equivalent and simpler method for solving integrable equations, especially soliton solutions [1014]. R-H approach [15-19] is extensively applied in lots of nonlinear PDEs, for example, the coupled $\mathrm{mKdV}$ equation [20], the generalized Sasa-Satsuma equation [21], the general coupled nonlinear Schrödinger equation [22], which plays a vital role in dealing with initial boundary value problems [23-25], discussing long-time asymptotic behavior [26] and investigating the lump solutions [27].
We find that construction of the Lax pair is critical for solving the R-H problem. However, there is no unified way to build Lax pair so far. In 1975, Wahlquist and Estabrook [28] proposed prolongation structures theory [29-31]. This method is one of the effective skills to verify whether the equation is integrable or not. The theory has been widely applied to many integrable equations, such as coupled $\mathrm{KdV}$ equation [32,33], coupled nonlinear Schröinger equation [34], and Heisenberg ferromagnet equation [35].

In this paper, we get Lax pair of NLST equation [36]

$$
i u_{t}+u_{x x}+2 u|u|^{2}-i a u^{2} \bar{u}_{x}-2 i b|u|^{2} u_{x}=0,
$$

via prolongation structures theory, where $\mathrm{a}$ and $\mathrm{b}$ are real parameters describing the measure of derivative cubic nonlinearity, the "-" denotes complex conjugation. Moreover, we formulate a R-H problem by the obtained Lax pair and get the $\mathrm{N}$-soliton solutions of (1). As we all know, the NLS equation

$$
i u_{t}+u_{x x}+2 u|u|^{2}=0
$$

is a vital model in physics, which plays a significant part in describing soliton propagation in water waves, nonlinear fiber optics plasma, etc. Similarly, system (1) can also describe 
different phenomena, such as collision and bound state. In particular, when setting $a=b=0$, (1) is reduced to classical NLS equation (2). For convenience, we set $a=b=1$ in what follows.

This paper is structured as follows. In Section 2, Lax pair related to (1) is obtained via prolongation structures theory. In Section 3, the analytical properties for an equivalent spectral problem are analyzed; besides, a R-H problem related to a newly introduced spatial matrix spectral problem is formulated. Through the formalism of $\mathrm{R}-\mathrm{H}$ problem, the construction of N-soliton solutions is discussed in Section 4. Finally, a few conclusions and some discussions are given.

\section{Prolongation Structures of the NLST Equation}

In this part, we study prolongation structures of equation

$$
i u_{t}+u_{x x}+2 u|u|^{2}-i u^{2} \bar{u}_{x}-2 i|u|^{2} u_{x}=0,
$$

which successfully gives the Lax pair of the NLST equation (3). The specific steps are as follows.

Firstly, we introduce an important proposition in the representation theory of Lie algebra.

Proposition 1 (see [37]). Let $X$ and $Y$ be two elements of Lie $g=s l(n+1, c)$, such that $[X, Y]=a Y,(a \neq 0)$ and $X \in$ range ad $Y$. Then we may identity $Y$ with $e_{ \pm}$and $X$ with $\pm(1 / 2) a h$, where $e_{ \pm}$are the nilpotent elements of $g$ and $h$ is the neutral element of $g$.

Secondly, a new series of independent variables for (3) are defined by $\bar{u}=p, \bar{u}_{x}=p_{x}=q, u_{x}=v$. Then (3) can be written as follows:

$$
\begin{aligned}
u_{x} & =v, \\
p_{x} & =q, \\
i u_{t}+v_{x}+2 u^{2} p-i u^{2} q-2 i u p v & =0, \\
-i p_{t}+q_{x}+2 p^{2} u+i p^{2} v+2 i u p q & =0 .
\end{aligned}
$$

And a set of differential 2-forms $I=\left\{\alpha_{1}, \alpha_{2}, \alpha_{3}, \alpha_{4}\right\}$ are defined on a differential manifold $M=\{x, t, u, v, p, q\}$, where

$$
\begin{aligned}
\alpha_{1}= & d u \Lambda d t+v d t \Lambda d x, \\
\alpha_{2}= & d p \Lambda d t+q d t \Lambda d x, \\
\alpha_{3}= & i d u \Lambda d x-d v \Lambda d t+2 u^{2} p d t \Lambda d x-i u^{2} q d t \Lambda d x \\
& -2 i u p v d t \Lambda d x, \\
\alpha_{4}= & -i d p \Lambda d x-d q \Lambda d t+2 p^{2} u d t \Lambda d x+i p^{2} v d t \Lambda d x \\
& +2 i u p q d t \Lambda d x .
\end{aligned}
$$

It is easy to testify the ideal is a closed ideal, that is $d I \subset I$, and limiting $I$ to solution manifold, we can get (3) and (4). Then, we introduce the differential 1-forms

$$
\omega^{i}=d y^{i}-F^{i}\left(u, v, p, q ; y^{i}\right) d x-G^{i}\left(u, v, p, q ; y^{i}\right) d t,
$$

where $F^{i}$ is linearly dependent on $y^{i}$ as well as $G^{i}$, that is $F^{i}=F_{j}^{i} y^{i}, G^{i}=G_{j}^{i} y^{i}$. For simplicity, $F_{j}^{i}$ is written as $F$ and $G_{j}^{i}$ is written as $G$ in what follows. According to the general theory of the exterior differential system, we obtain $\widetilde{I}=\left\{\alpha_{1}, \alpha_{2}, \alpha_{3}, \alpha_{4} ; \omega^{i}\right\}$ as a closed ideal, namely

$$
d \omega^{i}=\sum_{j=1}^{4}\left(f_{j}^{i} \alpha^{j}\right)+\eta_{j}^{i} \Lambda \omega^{i}
$$

where $f_{j}^{i}$ are functions to be ensured and $\eta^{j}$ is differential 1forms. Then, we obtain a suit of nonlinear PDEs of $F$ and $G$ :

$$
\begin{aligned}
& F_{v}=0 \\
& F_{q}=0 \\
& i G_{v}+F_{u}=0 \\
& i G_{q}-F_{p}=0 \\
& -G_{u} v-G_{p} q+2 u^{2} p G_{v}-i u^{2} q G_{v}-2 i u p v G_{v}+2 p^{2} u G_{q} \\
& \quad+i p^{2} v G_{q}+2 i u p q G_{q}+[F, G]=0,
\end{aligned}
$$

where $[F, G]=F G-G F$. Then set $F$ in (8) as follows:

$$
F=x_{0}+x_{1} u+x_{2} p
$$

where $x_{k}(k=0,1,2)$ are $2 \times 2$ matrices. Then, taking (9) into $i G_{v}+F_{u}=0, i G_{q}-F_{p}=0$, we can get a form of $G$

$$
G=i x_{1} v-i x_{2} q+H(u, p) \text {. }
$$

Substituting (10) into (8), we gain

$$
\begin{aligned}
& -H_{u}+2 u p x_{1}+p^{2} x_{2}+i\left[x_{0}, x_{1}\right]+i p\left[x_{2}, x_{1}\right]=0, \\
& -H_{p}+2 u p x_{2}+u^{2} x_{1}-i\left[x_{0}, x_{2}\right]-i u\left[x_{1}, x_{2}\right]=0,
\end{aligned}
$$

by collecting the coefficients of $v, q$. Setting $\left[x_{1}, x_{0}\right]=-x_{3}$, $\left[x_{2}, x_{1}\right]=-x_{5},\left[x_{0}, x_{2}\right]=x_{4}$, (11) can be written as

$$
\begin{aligned}
& H_{u}=2 u p x_{1}+i x_{3}-i p x_{5}+p^{2} x_{2}, \\
& H_{p}=2 u p x_{2}-i x_{4}-i u x_{5}+u^{2} x_{1} .
\end{aligned}
$$

Then we have

$$
\begin{aligned}
H(u, p)= & p^{2} u x_{2}+u^{2} p x_{1}+i u x_{3}-i p u x_{5}-i p x_{4} \\
& +H_{1} .
\end{aligned}
$$

Letting $H_{1}=x_{6}$, we finally obtain

$$
\begin{aligned}
F= & x_{0}+x_{1} u+x_{2} p \\
G= & i x_{1} v-i x_{2} q+p^{2} u x_{2}+u^{2} p x_{1}+i u x_{3}-i p u x_{5} \\
& -i p x_{4}+x_{6},
\end{aligned}
$$


where $L=\left\{x_{i}, i=0,1,2,3,4,5,6\right\}$ constitutes a continuation algebra and they have the following relationships:

$$
\begin{aligned}
2 i x_{1}+\left[x_{0}, x_{1}\right]-i\left[x_{1}, x_{5}\right] & =0, \\
-2 i x_{2}+\left[x_{0}, x_{2}\right]-i\left[x_{2}, x_{5}\right] & =0, \\
{\left[x_{0}, x_{5}\right]+\left[x_{1}, x_{4}\right]-\left[x_{2}, x_{3}\right] } & =0, \\
i\left[x_{0}, x_{3}\right]+\left[x_{1}, x_{6}\right] & =0, \\
{\left[x_{2}, x_{6}\right]-i\left[x_{0}, x_{4}\right] } & =0, \\
{\left[x_{1}, x_{3}\right] } & =0, \\
{\left[x_{2}, x_{4}\right] } & =0, \\
{\left[x_{0}, x_{6}\right] } & =0 .
\end{aligned}
$$

Finally, we embed continuation algebra $L$ into Lie algebra $s l(2, C)$. Through (15) and Proposition 1, we can get that $x_{1}$ and $x_{2}$ are nilpotent elements, and $x_{5}$ is neutral element, namely,

$$
x_{1}=\left(\begin{array}{ll}
0 & \lambda \\
0 & 0
\end{array}\right) \text {, }
$$

$$
\begin{aligned}
& F=\left(\begin{array}{cc}
i\left(\lambda^{2}-1\right) & \lambda u \\
-\lambda \bar{u} & -i\left(\lambda^{2}-1\right)
\end{array}\right), \\
& G=\left(\begin{array}{cc}
i \lambda^{2}|u|^{2}-2 i\left(\lambda^{2}-1\right)^{2} & i \lambda u_{x}+2 i u\left(i \lambda^{3}-i \lambda\right)+\lambda u^{2} \bar{u} \\
i \lambda \bar{u}_{x}-2 i \bar{u}\left(i \lambda^{3}-i \lambda\right)-\lambda \bar{u}^{2} u & -i \lambda^{2}|u|^{2}+2 i\left(\lambda^{2}-1\right)^{2}
\end{array}\right) .
\end{aligned}
$$

In summary, the Lax pair of (3) is

$$
\begin{aligned}
& \Psi_{x}=\left(\begin{array}{cc}
i\left(\lambda^{2}-1\right) & \lambda u \\
-\lambda \bar{u} & -i\left(\lambda^{2}-1\right)
\end{array}\right) \Psi, \\
& \Psi_{t}=\left(\begin{array}{cc}
i \lambda^{2}|u|^{2}-2 i\left(\lambda^{2}-1\right)^{2} & i \lambda u_{x}+2 i u\left(i \lambda^{3}-i \lambda\right)+\lambda u^{2} \bar{u} \\
i \lambda \bar{u}_{x}-2 i \bar{u}\left(i \lambda^{3}-i \lambda\right)-\lambda \bar{u}^{2} u & -i \lambda^{2}|u|^{2}+2 i\left(\lambda^{2}-1\right)^{2}
\end{array}\right) \Psi .
\end{aligned}
$$

\section{The Riemann-Hilbert Problem}

Before we formulate a R-H problem, we provide a definition of Riemann-Hilbert problem.

Definition 2 (Riemann-Hilbert problem). Let the contour $\Gamma$ be the union of a finite number of smooth and oriented curves on the Riemann sphere $\mathbb{C}$, such that $\mathbb{C} \backslash \Gamma$ has only a finite number of connected components. Let $J(\lambda)$ be a $2 \times 2$ matrix defined on the contour $\Gamma$. The $\operatorname{RHP}(\Gamma, J)$ is the problem of finding a $2 \times 2$ matrix-valued function $M(\lambda)$ that satisfies the following:

$$
\begin{aligned}
& x_{2}=\left(\begin{array}{cc}
0 & 0 \\
-\lambda & 0
\end{array}\right), \\
& x_{5}=\left(\begin{array}{cc}
-\lambda^{2} & 0 \\
0 & \lambda^{2}
\end{array}\right) .
\end{aligned}
$$

Then, we take (16) into (15) yielding

$$
\begin{aligned}
& x_{3}=\left(\begin{array}{cc}
0 & 2\left(i \lambda^{3}-i \lambda\right) \\
0 & 0
\end{array}\right), \\
& x_{4}=\left(\begin{array}{cc}
0 & 0 \\
2\left(i \lambda^{3}-i \lambda\right) & 0
\end{array}\right), \\
& x_{6}=\left(\begin{array}{cc}
-2 i\left(\lambda^{2}-1\right)^{2} & 0 \\
0 & 2 i\left(\lambda^{2}-1\right)^{2}
\end{array}\right) .
\end{aligned}
$$

Moreover, we obtain specific expressions for $F$ and $G$ :

(i) $M(\lambda)$ is analytic for all $\lambda \in \mathbb{C} \backslash \Gamma$ and extends continuously to the contour $\Gamma$;

(ii) $M_{+}(\lambda)=M_{-}(\lambda) J(\lambda), \lambda \in \Gamma$;

(iii) $M(\lambda) \longrightarrow I$, as $\lambda \longrightarrow \infty$.

The Lax pair of the NLST equation (3) reads

$$
\Psi_{x}=U \Psi=\left[i\left(\lambda^{2}-1\right) \Lambda+\lambda U_{1}\right] \Psi,
$$




$$
\begin{aligned}
\Psi_{t} & =V \Psi \\
& =\left[-2 i\left(\lambda^{2}-1\right)^{2} \Lambda-2 \lambda^{3} U_{1}+i \lambda^{2} U_{2}+\lambda U_{3}\right] \Psi,
\end{aligned}
$$

where $\Lambda=\operatorname{diag}(1,-1), \lambda \in \mathbb{C}$ is a spectral parameter,

$$
\begin{aligned}
U_{1} & =\left(\begin{array}{cc}
0 & u \\
-\bar{u} & 0
\end{array}\right), \\
U_{2} & =\left(\begin{array}{cc}
|u|^{2} & 0 \\
0 & -|u|^{2}
\end{array}\right), \\
U_{3} & =\left(\begin{array}{cc}
0 & i u_{x}+2 u+u^{2} \bar{u} \\
i \bar{u}_{x}-\bar{u}^{2} u-2 \bar{u} & 0
\end{array}\right),
\end{aligned}
$$

and the "-" represents complex conjugation.

In this part, the inverse scattering transforms of the NLST equation (3) are given by formulating a $\mathrm{R}-\mathrm{H}$ problem. The results will lay a foundation for the derivation of $N$-soliton solutions in Section 4.

We suppose that the potential $u$ is smooth enough and decays to zero fast when $x, t \longrightarrow \pm \infty$. In what follows, we treat $\Psi(x, t ; \lambda)$ as a matrix function. From (20a) and (20b), as $x, t \longrightarrow \pm \infty$, we notice that one possesses the asymptotic behavior: $\Psi \sim E=e^{i\left(\lambda^{2}-1\right) \Lambda x-2 i\left(\lambda^{2}-1\right)^{2} \Lambda t}$. Thus it is convenient to introduce a new matrix spectral function $J(x, t ; \lambda)$ which is defined as

$$
\Psi=J E .
$$

Inserting (22) into (20a) and (20b), the original form of Lax pair (20a) and (20b) becomes

$$
\begin{aligned}
& J_{x}=i\left(\lambda^{2}-1\right)[\Lambda, J]+V_{1} J, \\
& J_{t}=-2 i\left(\lambda^{2}-1\right)^{2}[\Lambda, J]+V_{2} J,
\end{aligned}
$$

with

$$
\begin{aligned}
& V_{1}=\left(\begin{array}{cc}
0 & \lambda u \\
-\lambda \bar{u} & 0
\end{array}\right), \\
& V_{2} \\
& =\left(\begin{array}{cc}
i \lambda^{2}|u|^{2} & i \lambda u_{x}-2 \lambda^{3} u+2 \lambda u+\lambda u^{2} \bar{u} \\
i \lambda \bar{u}_{x}+2 \lambda^{3} \bar{u}-2 \lambda \bar{u}-\lambda \bar{u}^{2} u & -i \lambda^{2}|u|^{2}
\end{array}\right),
\end{aligned}
$$

where $[\Lambda, J]=\Lambda J-J \Lambda$, and

$$
V_{1}^{\dagger}=-V_{1}
$$

where " $\dagger$ " represents the Hermitian of a matrix.

Let us now consider formulating a correlated $\mathrm{R}-\mathrm{H}$ problem with variable $x$. Firstly, in the scattering process, we introduce matrix Jost solutions $J_{ \pm}(x, \lambda)$ for spectral problem (23a)

$$
J_{ \pm}=\left(\left[J_{ \pm}\right]_{1},\left[J_{ \pm}\right]_{2}\right)
$$

which satisfy the asymptotic conditions

$$
\begin{aligned}
& J_{ \pm} \longrightarrow I, \\
& x \longrightarrow \pm \infty,
\end{aligned}
$$

respectively, where $I$ is a $2 \times 2$ unit matrix, each $\left[J_{ \pm}\right]_{k}(\mathrm{k}=1,2)$ represents the $k$-th column of the matrices $J_{+}$, respectively. The subscript of $J$ indicates at which end of the $x$-axis boundary conditions are set. Utilizing the parameter variation method and the boundary condition (28), the matrix spectral problem (23a) is transformed into the Volterra integral equations of $J_{ \pm}$

$$
\begin{aligned}
& J_{-}(x, \lambda)=\rrbracket+\int_{-\infty}^{x} e^{i\left(\lambda^{2}-1\right) \Lambda(x-y)} V_{1}(y) J_{-}(\lambda, y) \\
& \cdot e^{i\left(\lambda^{2}-1\right) \Lambda(y-x)} \mathrm{d} y, \\
& J_{+}(x, \lambda)=\rrbracket-\int_{x}^{+\infty} e^{i\left(\lambda^{2}-1\right) \Lambda(x-y)} V_{1}(y) J_{+}(\lambda, y) \\
& \cdot e^{i\left(\lambda^{2}-1\right) \Lambda(y-x)} \mathrm{d} y .
\end{aligned}
$$

Via a direct analysis of (29), due to the structure of the potential $V_{1}$, it is easy to see the second column of $J_{-}$involving the exponential factor $e^{i\left(\lambda^{2}-1\right)(x-y)}$, which, since $y<x$, decays when $\lambda \in \mathbb{C}_{+}=\{\lambda \mid \arg \lambda \in(0, \pi / 2) \cup(\pi, 3 \pi / 2)\}$. In addition, the first column of $J_{+}$contains the exponential factor $e^{-i\left(\lambda^{2}-1\right)(x-y)}$, which, since $y>x$, also decays when $\lambda \in \mathbb{C}_{+}$. Therefore, we believe these two columns are analytic for $\lambda \in \mathbb{C}_{+}$and continuous for $\lambda \in \mathbb{C}_{+} \cup \mathbb{R} \cup i \mathbb{R}$. Similarly, the first column of $J_{-}$and the second column of $J_{+}$also can be analytic for $\lambda \in \mathbb{C}_{-}=\{\lambda \mid \arg \lambda \in(\pi / 2, \pi) \cup(3 \pi / 2,2 \pi)\}$ and continuous for $\lambda \in \mathbb{C}_{-} \cup \mathbb{R} \cup i \mathbb{R}$.

Remark 3. This implementation of the inverse scattering transform for the NLST is very similar to that for the cubic NLS [38] except for a few key differences. The difference here is that one instead needs to distinguish between the upper and lower half planes of $\left(\lambda^{2}-1\right)$. And the other difference is the continuous of $\left(\lambda^{2}-1\right)$.

Notice that $\operatorname{tr}\left(V_{1}\right)=\operatorname{tr}\left(V_{2}\right)=0$, and we can use Abel's formula to get

$$
\operatorname{det} J(x, \lambda)=1, \quad \lambda \in \mathbb{R} \cup i \mathbb{R} .
$$

Then, through the asymptotic condition (28), we gain $\operatorname{det} J_{ \pm}=$ 1 for all $x$. Then, we introduce the notation $E(x, \lambda)=$ $e^{i\left(\lambda^{2}-1\right) \Lambda x}$. Since $\phi=J_{+} E$ is solution of $(20 \mathrm{a})$ and $\Phi=J_{-} E$ is solution as well, they must be linearly related, that is,

$$
J_{-} E=J_{+} E S(\lambda), \quad \lambda \in \mathbb{R} \cup i \mathbb{R},
$$

where

$$
S(\lambda)=\left(\begin{array}{ll}
s_{11} & s_{12} \\
s_{21} & s_{22}
\end{array}\right), \quad \lambda \in \mathbb{R} \cup i \mathbb{R},
$$

is the scattering matrix. Notice that $\operatorname{det} S(\lambda)=1$ because $\operatorname{det} J_{ \pm}=1$. 
Moreover, by analyzing the properties of $J_{-}$, we find $s_{11}$ accepts analytic extensions to $\mathbb{C}_{-}$and $s_{22}$ extends to $\mathbb{C}_{+}$ analytically.

The $\mathrm{R}-\mathrm{H}$ problem requires (3) to contain two matrix functions: one is analytic in $\mathbb{C}_{+}$, and the other is analytic in $\mathbb{C}_{-}$. We construct the first matrix function

$$
P^{+}(x, \lambda)=\left(\left[J_{+}\right]_{1},\left[J_{-}\right]_{2}\right)=J_{-} H_{2}+J_{+} H_{1},
$$

which is analytic in $\lambda \in \mathbb{C}_{+}$. Here, $H_{1}=\operatorname{diag}(1,0)$, $H_{2}=\operatorname{diag}(0,1)$. Furthermore, through the Volterra integral equations (29)-(30), we have

$$
\begin{aligned}
& P^{+}(x, \lambda) \longrightarrow I, \quad \lambda \in \mathbb{C}_{+} \rightarrow \infty, \\
&\left(\left[J_{-}\right]_{1},\left[J_{+}\right]_{2}\right) \longrightarrow I, \quad \lambda \in \mathbb{C}_{-} \longrightarrow \infty .
\end{aligned}
$$

In order to formulate the $\mathrm{R}-\mathrm{H}$ problem of (3), it is necessary to construct a matrix function $P^{-}$, which is analytic in $\mathbb{C}_{-}$for $\lambda$. Actually, we consider the inverse matrices of $J_{ \pm}$

$$
\begin{aligned}
& J_{-}^{-1}=\left(\begin{array}{l}
{\left[J_{-}^{-1}\right]_{1}} \\
{\left[J_{-}^{-1}\right]_{2}}
\end{array}\right), \\
& J_{+}^{-1}=\left(\begin{array}{l}
{\left[J_{+}^{-1}\right]_{1}} \\
{\left[J_{+}^{-1}\right]_{2}}
\end{array}\right),
\end{aligned}
$$

where $\left[J_{ \pm}^{-1}\right]_{k}(k=1,2)$ represent the $k$-th row of $J_{ \pm}^{-1}$, respectively. Moreover, $\left[J_{ \pm}^{-1}\right]_{k}$ satisfy the equation

$$
K_{x}=i\left(\lambda^{2}-1\right)[\Lambda, K]-K V_{1},
$$

which is called adjoint scattering equation of (23a). The matrix function $P^{-}$is introduced as

$$
P^{-}(x, \lambda)=\left(\begin{array}{l}
{\left[J_{+}^{-1}\right]_{1}} \\
{\left[J_{-}^{-1}\right]_{2}}
\end{array}\right)=H_{2} J_{-}^{-1}+H_{1} J_{+}^{-1},
$$

which is analytic in $\lambda \in \mathbb{C}_{-}$. With a similar process as $P^{+}$, we have

$$
\begin{aligned}
P^{-}(x, \lambda) & \longrightarrow I, \quad \lambda \in \mathbb{C}_{-} \rightarrow \infty, \\
\left(\left[J_{-}^{-1}\right]_{1},\left[J_{+}^{-1}\right]_{2}\right) & \longrightarrow I, \quad \lambda \in \mathbb{C}_{+} \longrightarrow \infty .
\end{aligned}
$$

We introduce

$$
\begin{aligned}
G^{+}(x, \lambda) & =\lim _{\mu \in \mathbb{C}_{+}, \mu \rightarrow \lambda} P^{+}(x, \mu), \\
\left(G^{-}\right)^{-1}(x, \lambda) & =\lim _{\mu \in \mathbb{C}_{-}, \mu \rightarrow \lambda} P^{-}(x, \mu), \quad \lambda \in \mathbb{R} \cup i \mathbb{R},
\end{aligned}
$$

related to

$$
G^{+}(x, \lambda)\left(G^{-}\right)^{-1}=G(x, \lambda)
$$

where

$$
\begin{aligned}
G(x, \lambda) & =E\left(H_{1}+H_{2} S\right)\left(H_{1}+S^{-1} H_{2}\right) E^{-1} \\
& =E\left(\begin{array}{cc}
1 & -s_{12} \\
s_{21} & 1
\end{array}\right) E^{-1} .
\end{aligned}
$$

Equations (43)-(44) accurately give a R-H problem of a correlation matrix. From (35) and (40), we get the asymptotic properties of the above $\mathrm{R}-\mathrm{H}$ problem

$$
P^{ \pm}(x, \lambda) \longrightarrow I, \lambda \longrightarrow \infty,
$$

and the canonical normalization condition

$$
G^{ \pm}(x, \lambda) \longrightarrow I, \quad \lambda \in \mathbb{R} \cup i \mathbb{R} \longrightarrow \infty
$$

\section{N-Soliton Solutions and Their Dynamics}

In this part, we derive $N$-soliton solutions for NLST equation (3). As is well known that R-H problem with zeros produces soliton solutions, the uniqueness of the solution of associated $\mathrm{R}-\mathrm{H}$ problem defined in (43) and the zeros of $\operatorname{det} P^{ \pm}$are specified in their analytic domains, and the structure of $\operatorname{ker} P^{ \pm}$at these zeros can be determined. From the definitions of equations (34) and (39) as well as the scattering relation (32), one gets

$$
\begin{aligned}
& \operatorname{det} P^{+}(x, \lambda)=s_{22}(\lambda), \\
& \operatorname{det} P^{-}(x, \lambda)=\widehat{s}_{22}(\lambda),
\end{aligned}
$$

where $\widehat{s}_{22}(\lambda)=\left(s^{-1}\right)_{22}=s_{11}$.

Let us assume that $s_{22}$ has $N$ zeros $\left\{\lambda_{k} \in \mathbb{C}_{+}, 1 \leq k \leq N\right\}$ and $\widehat{s}_{22}$ has $N$ zeros $\left\{\widehat{\lambda}_{k} \in \mathbb{C}_{-}, 1 \leq k \leq N\right\}$. For convenience, we suppose that all zeros $\left(\lambda_{k}, \hat{\lambda}_{k}\right)$ are simple zeros of $\left(s_{22}, \widehat{s}_{22}\right)$. In what follows, each of ker $P^{+}\left(\lambda_{k}\right), 1 \leq k \leq N$, which only includes a single column vector $v_{k}$; each of $\operatorname{ker} P^{-}\left(\hat{\lambda}_{k}\right), 1 \leq$ $k \leq N$, which only includes a single row vector $\widehat{v}_{k}$, namely,

$$
\begin{aligned}
& P^{+}\left(\lambda_{k}\right) v_{k}=0, \quad 1 \leq k \leq N \\
& \widehat{v}_{k} P^{-}\left(\hat{\lambda}_{k}\right)=0, \quad 1 \leq k \leq N .
\end{aligned}
$$

A key step for solving soliton solutions is to calculate the potential matrix $V_{1}$ through $P^{ \pm}(x, \lambda)$. Notice that $P^{+}$is the solution of the spectral problem (23a); consequently, we consider the asymptotic expansion of $P^{+}$,

$$
P^{+}(x, \lambda)=I+\lambda^{-1} P_{1}^{+}(x)+\lambda^{-2} P_{2}^{+}(x)+o\left(\lambda^{-3}\right),
$$

$$
\lambda \longrightarrow \infty \text {, }
$$

submitting (49) into (23a) and comparing $o(\lambda)$ term, we obtain

$$
U_{1}=-i\left[\Lambda, P_{1}^{+}\right]=\left(\begin{array}{cc}
0 & -2 i\left(P_{1}^{+}\right)_{12} \\
2 i\left(P_{1}^{+}\right)_{21} & 0
\end{array}\right) .
$$

Thus, a direct calculation shows that the potentials $u$ is represented as

$$
u=-2 i\left(P_{1}^{+}\right)_{12} \text {. }
$$

The potential matrix $U_{1}$ has symmetry property, which produces in scattering matrix and Jost functions. In addition, 
the scattering equation (23a) has Hermitian property and we get

$$
\left(J_{ \pm}^{\dagger}\right)_{x}=i\left(\bar{\lambda}^{2}-1\right)\left[\Lambda, J_{ \pm}^{\dagger}\right]-J_{ \pm}^{\dagger} V_{1} .
$$

Notice that $J_{ \pm}^{\dagger}(x, \bar{\lambda})$ meet the adjoint scattering equation (38); recalling $J_{ \pm}^{-1}$ also meets (38), and using the boundary conditions (28) we obtain

$$
J_{ \pm}^{\dagger}(x, \bar{\lambda})=J_{ \pm}^{-1}(x, \lambda)
$$

Through this involution property and definitions of (34) as well as (39) for $P^{ \pm}$, we find the involution property is also fit for the analytic solutions $P^{ \pm}$:

$$
\left(P^{+}\right)^{\dagger}(\bar{\lambda})=P^{-}(\lambda)
$$

Furthermore, from the scattering relationship (32) between $J_{+}$and $J_{-}$, the involution property also applies to $S$ :

$$
S^{\dagger}(\bar{\lambda})=S^{-1}(\lambda) \text {. }
$$

In view of the involution property, we gain the relation

$$
\widehat{\lambda}_{k}=\bar{\lambda}_{k}
$$

for the zeros of $s_{22}(\lambda)$ and $\widehat{s}_{22}(\lambda)$. Without loss of generality, taking equations (48a), (54), (56), we have

$$
\widehat{v}_{k}=v_{k}^{\dagger} \text {. }
$$

The derived special $\mathrm{R}-\mathrm{H}$ problem is solved by referring to references and the results can be expressed as

$$
\begin{aligned}
& P^{+}(\lambda)=I-\sum_{k, l=1}^{N} \frac{v_{k}\left(M^{-1}\right)_{k l} \widehat{v}_{l}}{\lambda-\widehat{\lambda}_{l}}, \\
& P^{-}(\lambda)=I+\sum_{k, l=1}^{N} \frac{v_{k}\left(M^{-1}\right)_{k l} \widehat{v}_{l}}{\lambda-\lambda_{k}},
\end{aligned}
$$

$M$ is an $N \times N$ matrix given by

$$
\begin{aligned}
M & =\left(M_{k l}\right)_{N \times N}, \\
M_{k l} & =\frac{\widehat{v}_{k} v_{l}}{\widehat{\lambda}_{k}-\lambda_{l}},
\end{aligned}
$$

$$
1 \leq k, l \leq N
$$

Notice that zeros $\lambda_{k}$ and $\hat{\lambda}_{k}$ are constants; that is, spatial variable $x$ and time variable $t$ are independent. Thus, it is easy to determine the spatial and temporal evolution of vectors $v_{k}(x, t)$ and $\widehat{v}_{k}(x, t)(1 \leq k \leq N)$. We calculate the derivatives of $x$ on both sides of equation $P^{+}\left(\lambda_{k}\right) v_{k}=0$. Taking advantage of equation (23a), one gets

$$
P^{+}\left(x, \lambda_{k}\right)\left(\frac{d v_{k}}{d x}-i\left(\lambda^{2}-1\right) \Lambda v_{k}\right)=0, \quad 1 \leq k \leq N
$$

thus

$$
\frac{d v_{k}}{d x}=i\left(\lambda^{2}-1\right) \Lambda v_{k}, \quad 1 \leq k \leq N
$$

In a completely similar way, the time dependence of $v_{k}$ :

$$
\frac{d v_{k}}{d t}=-2 i\left(\lambda^{2}-1\right)^{2} \Lambda v_{k}, \quad 1 \leq k \leq N,
$$

can be determined by matrix spectral problem (20b). Summing up, we have

$$
\begin{aligned}
& v_{k}(x, t)=e^{i\left(\lambda_{k}^{2}-1\right) \Lambda x-2 i\left(\lambda_{k}^{2}-1\right)^{2} \Lambda t} v_{k_{0}}, \quad 1 \leq k \leq N, \\
& \widehat{v}_{k}(x, t)=\widehat{v}_{k_{0}} e^{-i\left(\widehat{\lambda}_{k}^{2}-1\right) \Lambda x+2 i\left(\widehat{\lambda}_{k}^{2}-1\right)^{2} \Lambda t}, \quad 1 \leq k \leq N,
\end{aligned}
$$

where $v_{k 0}$ is an arbitrary constant column vector, and $\widehat{v}_{k 0}$ is an arbitrary constant row vector.

Finally, from (58a) and (58b), we obtain

$$
P_{1}^{+}=-\sum_{k, l=1}^{N} v_{k}\left(M^{-1}\right)_{k l} \widehat{v}_{l}
$$

and from (51), the general N-soliton solutions of NLST equation read

$$
u=2 i\left(\sum_{k, l=1}^{N} v_{k}\left(M^{-1}\right)_{k l} \widehat{v}_{l}\right)_{12}
$$

where vectors $v_{k}$ are given by (63), $\widehat{v}_{l}=v_{l}^{\dagger}$, and matrix $M$ is given by (59). In order to get the soliton solutions of the NLST equation (3) conveniently, we let $v_{l 0}=\left(\alpha_{l}, \beta_{l}\right)^{T}$. Furthermore, we introduce the notation $\theta_{k}=i\left(\lambda_{k}^{2}-1\right) x-2 i\left(\lambda_{k}^{2}-1\right)^{2} t, 1 \leq$ $k \leq N$. Then the above solution $u$ is expressed explicitly as

$$
u(x, t)=2 i \sum_{k, l=1}^{N} \alpha_{k} \bar{\beta}_{l} e^{\theta_{k}-\bar{\theta}_{l}}\left(M^{-1}\right)_{k l}
$$

where

$$
M_{k l}=\frac{1}{\bar{\lambda}_{k}-\lambda_{l}}\left[\bar{\alpha}_{k} \alpha_{l} e^{\left(\bar{\theta}_{k}+\theta_{l}\right)}+\bar{\beta}_{k} \beta_{l} e^{-\bar{\theta}_{k}-\theta_{l}}\right] .
$$

4.1. Single-Soliton Solutions. We set $N=1$ in equation (67), the single-soliton solution reads

$$
u(x, t)=2 i\left(\bar{\lambda}_{1}-\lambda_{1}\right) \frac{\alpha_{1} \bar{\beta}_{1} e^{\theta_{1}-\bar{\theta}_{1}}}{|\beta|^{2} e^{-\left(\theta_{1}+\bar{\theta}_{1}\right)}+\left|\alpha_{1}\right|^{2} e^{\theta_{1}+\bar{\theta}_{1}}} .
$$

Let $\lambda_{1}=a+i b, \alpha_{1}=e^{-2 a b x_{0}+i \sigma_{0}}, \beta_{1}=1$, where $a, b$ are the real and imaginary of $\lambda_{1}$, and $x_{0}, \sigma_{0}$ are real parameters. Then, solution (67) is represented as

$$
\begin{aligned}
& u(x, t)=2 b \operatorname{sech}\left\{-4 a b\left[x+4\left(1-a^{2}+b^{2}\right) t\right]-x_{0}\right\} \\
& \cdot \exp \left\{2 i\left(a^{2}-b^{2}-1\right) x\right. \\
& \left.-4 i\left[\left(a^{2}-b^{2}-1\right)^{2}-4 a^{2} b^{2}\right] t+i \sigma_{0}\right\} .
\end{aligned}
$$




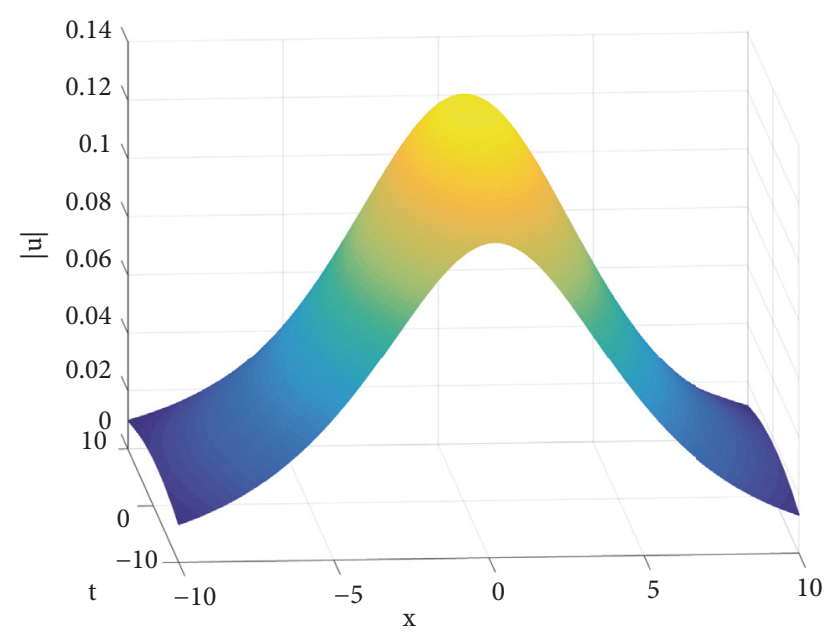

FIgURE 1: Modulus of single-soliton $u(x, t)$ in (70) with the parameters chosen as $a=1, b=-0.07, x_{0}=0.1, \sigma_{0}=0.1$.

Solution (70) is a solitary wave in the NLST equation (1). It is easy to see that the shape of the amplitude function $|u(x, t)|$ is hyperbolic secant, the peak amplitude is $2 b$, and the velocity is $-4\left(1-a^{2}+b^{2}\right)$. The phase of this solution depends linearly on time $t$ and space $x$. In addition, the parameters $x_{0}$ and $\sigma_{0}$ represent the initial position and the phase of the solitary wave, respectively. Notice that the soliton power depends on $b$ only; therefore, it does not change after soliton collisions. The solution is shown graphically in Figure 1.

Remark 4. One can see that the single-soliton solution (70) of the NLST equation and the single-soliton solution of the cubic NLS are different. From the difference in the Lax pair, one can follow through to see that eigenvalues of the cubic NLS (I will call them $k$ ) corresponding to $\left(\lambda^{2}-1\right)$ in this NLST. Thus since $k=\xi+i \eta$ in the cubic NLS corresponds to a pulse width of $2 \eta$ and velocity of $-4 \xi$, in the NLST we have $\lambda=a+i b$ corresponding to $\xi+i \eta=(a+i b)^{2}-1=\left(a^{2}-b^{2}-1\right)+2 i a b$, so the pulse width will be $-4 a b$ and the velocity will be $-4(1-$ $\left.a^{2}+b^{2}\right)$.

4.2. Two-Soliton Solutions. We set $N=2$ in formula (67), and the two-soliton solutions also can be expressed explicitly, while it is quite complicated. Using algebraic theory, twosoliton solutions are represented as

$$
u(x, t)=-2 i \frac{\operatorname{det} F}{\operatorname{det} M}
$$

where

$$
F=\left[\begin{array}{cccc}
0 & \alpha_{1} e^{\theta_{1}} & \cdots & \alpha_{N} e^{\theta_{N}} \\
\bar{\beta}_{1} e^{-\bar{\theta}_{1}} & M_{11} & \cdots & M_{1 N} \\
\vdots & \vdots & \ddots & \vdots \\
\bar{\beta}_{N} e^{-\bar{\theta}_{N}} & M_{N 1} & \cdots & M_{N N}
\end{array}\right]
$$

Equation (71) is read as

$$
\begin{gathered}
u(x, t)=-2 i \frac{-\alpha_{1} \bar{\beta}_{1} e^{\theta_{1}-\bar{\theta}_{1}} M_{22}+\alpha_{2} \bar{\beta}_{1} e^{\theta_{2}-\bar{\theta}_{1}} M_{21}}{|M|} \\
\cdot \frac{\alpha_{1} \bar{\beta}_{2} e^{\theta_{1}-\bar{\theta}_{2}} M_{12}-\alpha_{2} \bar{\beta}_{2} e^{\theta_{2}-\bar{\theta}_{2}} M_{11}}{|M|},
\end{gathered}
$$

where

$$
\begin{aligned}
& M_{11}=\frac{\left|\alpha_{1}\right|^{2} e^{\bar{\theta}_{1}+\theta_{1}}+\left|\beta_{1}\right|^{2} e^{-\bar{\theta}_{1}-\theta_{1}}}{-2 i b_{1}}, \\
& M_{12}=\frac{\bar{\alpha}_{1} \alpha_{2} e^{\bar{\theta}_{1}+\theta_{2}}+\bar{\beta}_{1} \beta_{2} e^{-\bar{\theta}_{1}-\theta_{2}}}{\left(\alpha_{1}-\alpha_{2}\right)-i\left(b_{1}+b_{2}\right)}, \\
& M_{21}=\frac{\bar{\alpha}_{2} \alpha_{1} e^{\bar{\theta}_{2}+\theta_{1}}+\bar{\beta}_{2} \beta_{1} e^{-\bar{\theta}_{2}-\theta_{1}}}{\left(\alpha_{2}-\alpha_{1}\right)-i\left(b_{1}+b_{2}\right)}, \\
& M_{22}=\frac{\left|\alpha_{2}\right|^{2} e^{\bar{\theta}_{2}+\theta_{2}}+\left|\beta_{2}\right|^{2} e^{-\bar{\theta}_{2}-\theta_{2}}}{-2 i b_{2}} .
\end{aligned}
$$

Denoting $\lambda_{k}=a_{k}+i b_{k}, k=1,2, a_{k}$ is the real part of $\lambda_{k}$ and $b_{k}$ is the imaginary part of $\lambda_{k}$. Two cases are given below: one case is $a_{1} \neq a_{2}$, which means that the two solitons have different velocities, and they form a collision, and the other case is $a_{1}=a_{2}$, which means that the two solitons have same velocities, and they form a bound state.

Case I. We choose $\lambda_{1}=1.5+0.55 i, \lambda_{2}=1+0.5 i$.

In this case, when $t \longrightarrow-\infty$, this solution consists of two single-solitons that are far apart and move toward each other. They interact when they collide. When $t \longrightarrow+\infty$, these solitons reappear from the interaction without any change in shape and velocity. The two-soliton solutions are shown graphically in Figure 2.

Case II. We choose $\lambda_{1}=1+0.35 i, \lambda_{2}=1+0.05 i$.

In this case, we assume $a_{1}=a_{2}$, the two solitons have same velocity; therefore, they will stay together and form a bound state. In addition, the amplitude function $|u(x, t)|$ has periodic oscillations with time as shown in Figure 3.

In summary, we obtained the $\mathrm{N}$-soliton solutions (67) by the $\mathrm{R}-\mathrm{H}$ approach. In addition, as an example, the expressions of the single-soliton solutions (69) and the two-soliton solutions (71) are given explicitly. Moreover, we discuss the dynamic behaviors of two solitons. By giving two examples, two different phenomena are shown, one is $a_{1} \neq a_{2}$, which describes a collision; the other is $a_{1}=a_{2}$, which describes a bound state. Similarly, we can get three-soliton solutions, four-soliton solutions, etc. It is also interesting to study their dynamic behaviors in the future.

\section{Conclusion}

In this work, a new integrable NLST equation is investigated via prolongation structures theory and $\mathrm{R}-\mathrm{H}$ approach. We 


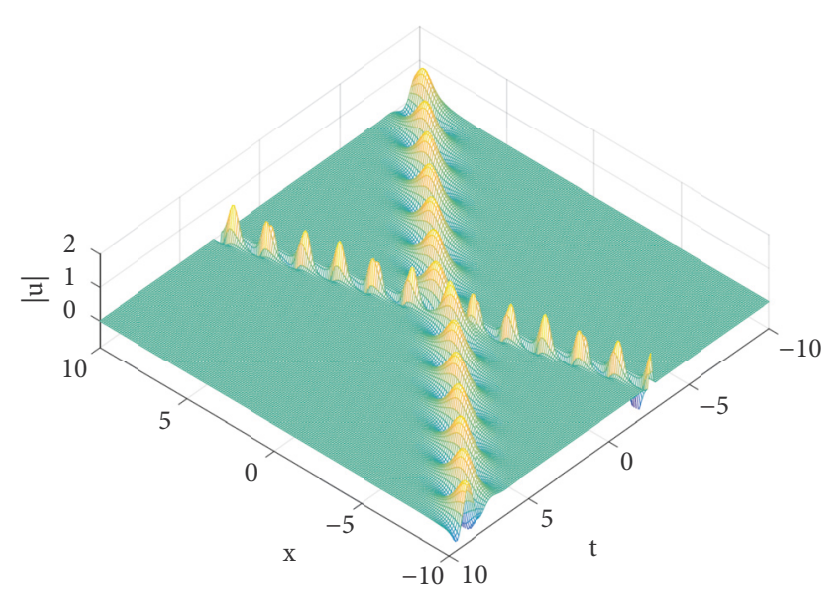

Figure 2: Collision modulus of two-soliton $u(x, t)$ in (71) with the parameters chosen as $a_{1}=1.5, b_{1}=0.55, a_{2}=1, b_{2}=0.5, \alpha_{1}=$ 0.4 $(1+i), \beta_{1}=0.4(1-i), \alpha_{2}=0.4(1-i), \beta_{2}=0.4(1+i)$.

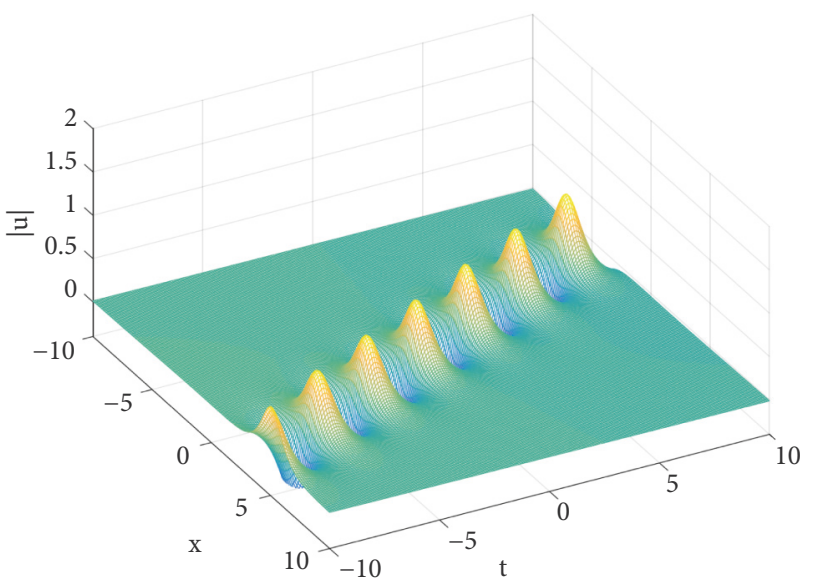

FIGURE 3: Bound state modulus of two-soliton $u(x, t)$ in (71) with the parameters chosen as $a_{1}=1, b_{1}=0.35, a_{2}=1, b_{2}=0.05$, $\alpha_{1}=\sqrt{2.5}(1+i), \beta_{1}=\sqrt{2.5}(1-i), \alpha_{2}=\sqrt{2.5}(1-i), \beta_{2}=\sqrt{2.5}(1+i)$.

apply the prolongation structures theory to the NLST equation; by discussing the continuation algebra of the NLST equation, a $2 \times 2$ Lax pair is successfully derived. Then, we begin with the spectral analysis of the Lax pair and formulate a $\mathrm{R}-\mathrm{H}$ problem. Through a specific $\mathrm{R}-\mathrm{H}$ problem with vanishing scattering coefficient, which is under the condition of the reflection-less case, $\mathrm{N}$-soliton solutions are obtained explicitly. In addition, we present a few key differences between the NLST equation and the cubic NLS equation in the implementation of inverse scattering transform. Moreover, we analyze the dynamic behaviors between two solitons, which describe different phenomena, such as collision, bound state, and the dynamic behaviors of the one-soliton solution and two-soliton solutions are shown by graphs, respectively.

Recently, we notice that there are many other approaches to obtain exact solutions in the field of integrable systems, like Hirota's bilinear method [39], Darboux transformation method [40], Wronskian technique [41], Frobenius integrable decompositions [42], etc. [43]. Thus we consider whether the NLST equation can be solved with these approaches. These questions will be discussed in our future work.

\section{Data Availability}

No data were used to support this study.

\section{Conflicts of Interest}

The authors declare that there are no conflicts of interest regarding the publication of this paper.

\section{Acknowledgments}

This work was supported by the Shandong Provincial Natural Science Foundation (Grant No. ZR2019QD018), National Natural Science Foundation of China (Grant No. 61602188), and Scientific Research Foundation of Shandong University of Science and Technology for Recruited Talents (Grant Nos. 2017RCJJ068 and 2017RCJJ069).

\section{References}

[1] W. X. Ma, H. Wu, and J. He, "Partial differential equations possessing Frobenius integrable decompositions," Physics Letters A, vol. 364, no. 1, pp. 29-32, 2007.

[2] X. Wang, Y. Fang, and H. Dong, "Component-trace identity for Hamiltonian structure of the integrable couplings of the Giachetti-Johnson (GJ) hierarchy and coupling integrable couplings," Communications in Nonlinear Science and Numerical Simulation, vol. 16, no. 7, pp. 2680-2688, 2011.

[3] L. Zhang, Y. Wang, C. M. Khalique, and Y. Bai, "Peakon and cuspon solutions of a generalized Camassa-Holm-Novikov equation," Journal of Applied Analysis and Computation, vol. 8, no. 6, pp. 1938-1958, 2018.

[4] Y. Liu, H. Dong, and Y. Zhang, "Solutions of a discrete integrable hierarchy by straightening out of its continuous and discrete constrained flows," Analysis and Mathematical Physics, vol. 9, pp. 465-481, 2019.

[5] M. Guo, H. Dong, J. Liu, and H. Yang, "The time-fractional mZK equation for gravity solitary waves and solutions using sech-tanh and radial basis function method," Nonlinear Analysis: Modelling and Control, vol. 24, no. 1, 19 pages, 2019.

[6] M. Tao and H. Dong, "Algebro-geometric solutions for a discrete integrable equation," Discrete Dynamics in Nature and Society, vol. 2017, 9 pages, 2017.

[7] M. Tao, N. Zhang, D. Gao, and H. Yang, "Symmetry analysis for three-dimensional dissipation Rossby waves," Advances in Difference Equations, vol. 300, 2018.

[8] M. J. Ablowitz and Z. H. Musslimani, "Inverse scattering transform for the integrable nonlocal nonlinear Schrödinger equation," Nonlinearity, vol. 29, no. 3, pp. 915-946, 2016.

[9] H. C. Morris, "Prolongation structures and a generalized inverse scattering problem," Journal of Mathematical Physics, vol. 17, no. 10, pp. 1867-1869, 1976.

[10] H. Yang, J. Sun, and C. Fu, "Time-fractional Benjamin-Ono equation for algebraic gravity solitary waves in baroclinic 
atmosphere and exact multi-soliton solution as well as interaction," Communications in Nonlinear Science and Numerical Simulation, vol. 71, pp. 187-201, 2019.

[11] M. Guo, C. Fu, Y. Zhang, J. Liu, and H. Yang, "Study of ionacoustic solitary waves in a magnetized plasma using the threedimensional time-space fractional Schamel-KdV equation," Complexity, vol. 2018, Article ID 6852548, 2018.

[12] M. Han, L. Zhang, Y. Wang, and C. M. Khalique, "The effects of the singular lines on the traveling wave solutions of modified dispersive water wave equations," Nonlinear Analysis: Real World Applications, vol. 47, pp. 236-250, 2019.

[13] W. Q. Peng, S. F. Tian, T. T. Zhang, and Y. Fang, "Rational and semi-rational solutions of a nonlocal $(2+1)$-dimensional nonlinear Schrodinger equation," Mathematical Methods in the Applied Sciences, 2019.

[14] H. W. Yang, M. Guo, and H. He, "Conservation laws of spacetime fractional mZK equation for Rossby solitary waves with complete Coriolis force," International Journal of Nonlinear Sciences and Numerical Simulation, vol. 20, pp. 17-32, 2018.

[15] V. S. Shchesnovich and J. Yang, "General soliton matrices in the Riemann-Hilbert problem for integrable nonlinear equations," Journal of Mathematical Physics, vol. 44, no. 10, pp. 4604-4639, 2003.

[16] D.-S. Wang, D.-J. Zhang, and J. Yang, "Integrable properties of the general coupled nonlinear Schrödinger equation," Journal of Mathematical Physics, vol. 51, no. 2, 17 pages, 2010.

[17] Z. Kang, T. Xia, and X. Ma, "Multi-soliton solutions for the coupled modified nonlinear Schrödinger equations via Riemann-Hilbert approach," Chinese Physics B, vol. 27, no. 7, p. 070201, 2018.

[18] X. G. Geng and H. Nie, "N solutions for a derivative nonlinear Schrodinger-type equation via Riemann-Hilbert approach," Mathematical Methods in the Applied Sciences, vol. 41, pp. 1653$1660,2018$.

[19] Y. Lin, H. Dong, and Y. Fang, "N-Soliton Solutions for the NLS-Like Equation and Perturbation Theory Based on the Riemann-Hilbert Problem," Symmetry, vol. 11, no. 6, p. 826, 2019.

[20] W. X. Ma, "Riemann-Hilbert problems and N-soliton solutions for a coupled mKdV system," Journal of Geometry and Physics, vol. 132, pp. 45-54, 2018.

[21] X. Geng and J. Wu, "Riemann-Hilbert approach and $N$ soliton solutions for a generalized Sasa-Satsuma equation," Wave Motion. An International Journal Reporting Research on Wave Phenomena, vol. 60, pp. 62-72, 2016.

[22] D.-S. Wang, D.-J. Zhang, and J. Yang, "Integrable properties of the general coupled nonlinear Schrödinger equation," Journal of Mathematical Physics, vol. 51, no. 2, Article ID 023510, 17 pages, 2010.

[23] J. Xu and E. Fan, "The initial-boundary value problem for the ostrovsky-vakhnenko equation on the half-line," Mathematical Physics, Analysis and Geometry, vol. 19, no. 3, p. 17, 2016.

[24] B. Hu, T. Xia, and W. Ma, "Riemann-Hilbert approach for an initial-boundary value problem of the two-component modified Korteweg-de Vries equation on the half-line," Applied Mathematics and Computation, vol. 332, pp. 148-159, 2018.

[25] T. Liu and H. Dong, "The prolongation structure of the modified nonlinear schrödinger equation and its initial-boundary value problem on the half line via the riemann-hilbert approach," Mathematics, vol. 7, no. 2, 2019.
[26] J. Xu, E. G. Fan, and Y. Chen, "Long-time asymptotic for the derivative nonlinear Schroinger equation with step-like initial value," Mathematical Physics, Analysis and Geometry, vol. 16, pp. 253-288, 2013.

[27] Y. Zhang, H. Dong, X. Zhang, and H. Yang, "Rational solutions and lump solutions to the generalized (3+1)-dimensional Shallow Water-like equation," Journal of Computers \& Mathematics with Applications, vol. 73, no. 2, pp. 246-252, 2017.

[28] H. D. Wahlquist and F. B. Estabrook, "Prolongation structures of nonlinear evolution equations," Journal of Mathematical Physics, vol. 16, pp. 1-7, 1975.

[29] H. C. Morris, "A prolongation structure for the AKNS system and its generalization," Journal of Mathematical Physics, vol. 18, no. 3, pp. 533-536, 1977.

[30] D. Wang, Y. Ma, and X. Li, "Prolongation structures and matter-wave solitons in $\mathrm{F}=1$ spinor Bose-Einstein condensate with time-dependent atomic scattering lengths in an expulsive harmonic potential," Communications in Nonlinear Science and Numerical Simulation, vol. 19, no. 10, pp. 3556-3569, 2014.

[31] Z. Yan and C. Li, "Fermionic covariant prolongation structure theory for a (2+1)-dimensional super nonlinear evolution equation," International Journal of Geometric Methods in Modern Physics, vol. 15, no. 07, p. 1850114, 2018.

[32] Y. Jia and W. Duan, “The Prolongation Structure of a Coupled Kdv Equation," Acta Mathematicae Applicatae Sinica, vol. 34, no. 2, pp. 430-437, 2018.

[33] Y. Cao and D. Wang, "Prolongation structures of a generalized coupled Korteweg-de Vries equation and Miura transformation," Communications in Nonlinear Science and Numerical Simulation, vol. 15, no. 9, pp. 2344-2349, 2010.

[34] D. Wang, S. Yin, Y. Tian, and Y. Liu, "Integrability and bright soliton solutions to the coupled nonlinear Schrödinger equation with higher-order effects," Applied Mathematics and Computation, vol. 229, pp. 296-309, 2014.

[35] W.-Z. Zhao, Y.-Q. Bai, and K. Wu, "Generalized inhomogeneous Heisenberg ferromagnet model and generalized nonlinear Schrödinger equation," Physics Letters A, vol. 352, no. 1-2, pp. 64-68, 2006.

[36] M. Wadati, K. Konno, and Y. Ichikawa, "Lax representation for the generalized nonlinear Schrodinger equation," Journal of the Physical Society of Japan, vol. 24, p. 1965, 1979.

[37] J. E. Humphreys, Introduction to Lie Algebras and Representation Theory, Springer, New York, NY, USA, 1972.

[38] S. Cuccagna and D. E. Pelinovsky, "The asymptotic stability of solitons in the cubic NLS equation on the line," Applicable Analysis: An International Journal, vol. 93, no. 4, pp. 791-822, 2012.

[39] W.-X. Ma and Y. Zhou, "Lump solutions to nonlinear partial differential equations via Hirota bilinear forms," Journal of Differential Equations, vol. 264, no. 4, pp. 2633-2659, 2018.

[40] N. Zhang, T. Xia, and Q. Jin, "N-Fold Darboux transformation of the discrete Ragnisco-Tu system," Advances in Difference Equations, vol. 2018, no. 1, 2018.

[41] S. Jia, Y. Gao, C. Ding, and G. Deng, "Solitons for a $(2+1)$ dimensional Sawada-Kotera equation via the Wronskian technique," Applied Mathematics Letters, vol. 74, pp. 193-198, 2017.

[42] Y. Fang, H. Dong, Y. Hou, and Y. Kong, "Frobenius integrable decompositions of nonlinear evolution equations with modified term," Applied Mathematics and Computation, vol. 226, no. 1, pp. 435-440, 2014. 
[43] B. Song and Y. Kong, "Solution to PSPACE-complete problem using p systems with active membranes with time-freeness," Mathematical Problems in Engineering, vol. 2019, 8 pages, 2019. 


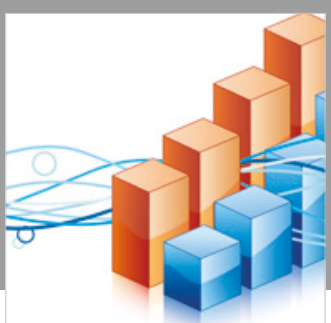

Advances in

Operations Research

\section{-n-m}
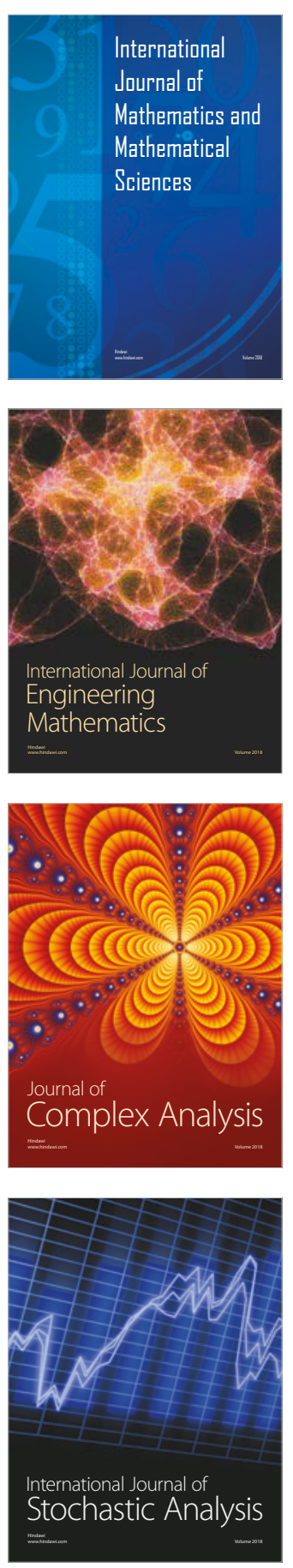
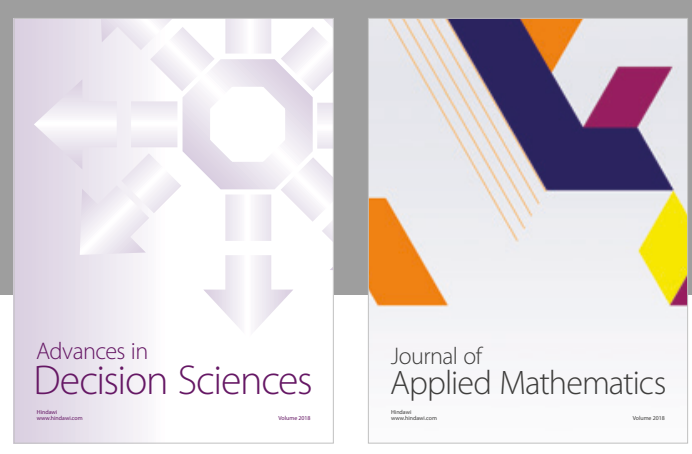

Journal of

Applied Mathematics
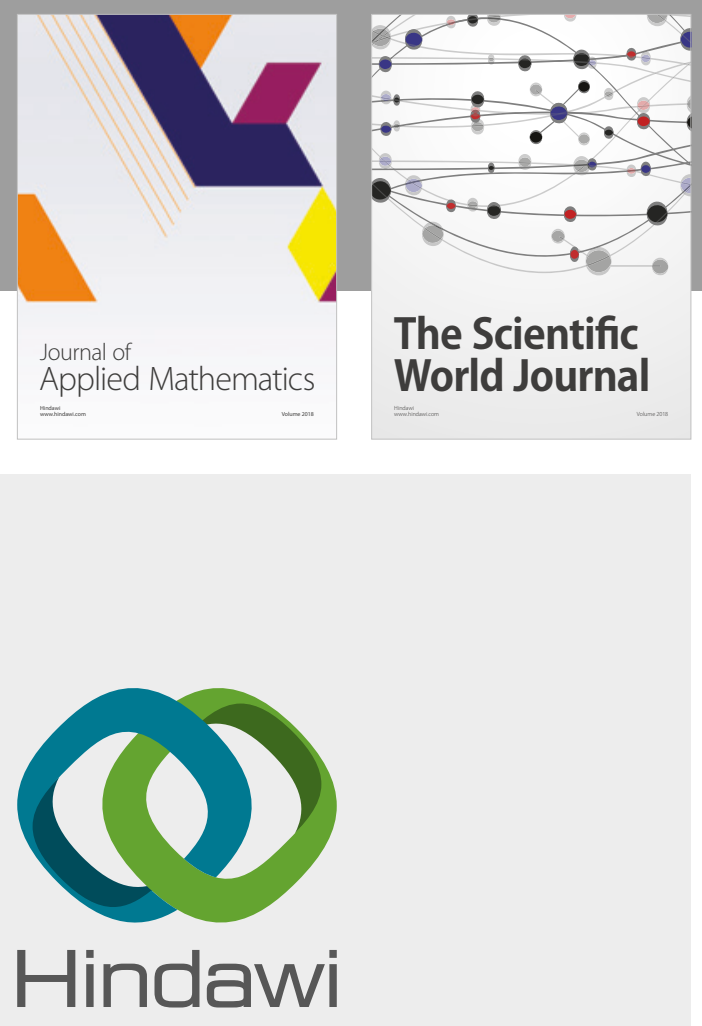

Submit your manuscripts at

www.hindawi.com

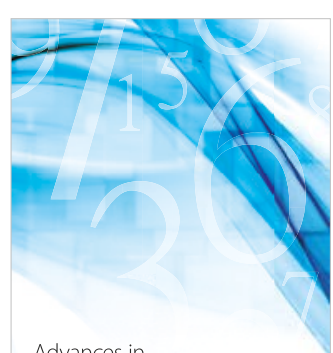

Advances in
Numerical Analysis
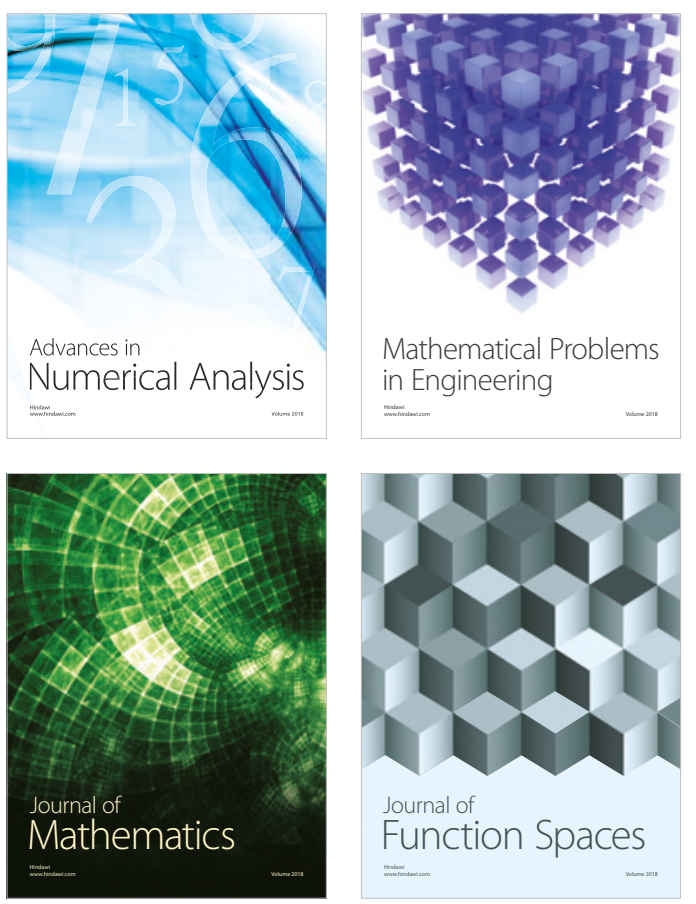

Mathematical Problems in Engineering

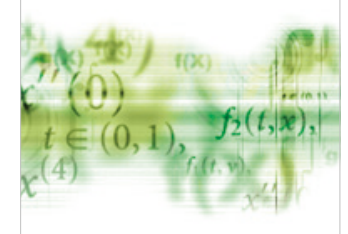

International Journal of

Differential Equations

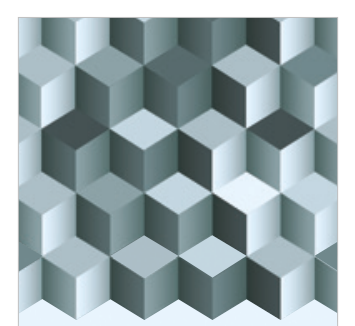

Journal of

Function Spaces

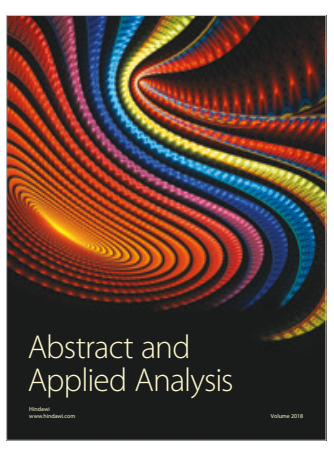

The Scientific

World Journal

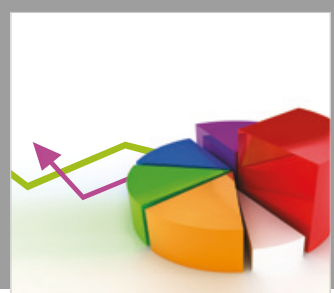

Journal of

Probability and Statistics
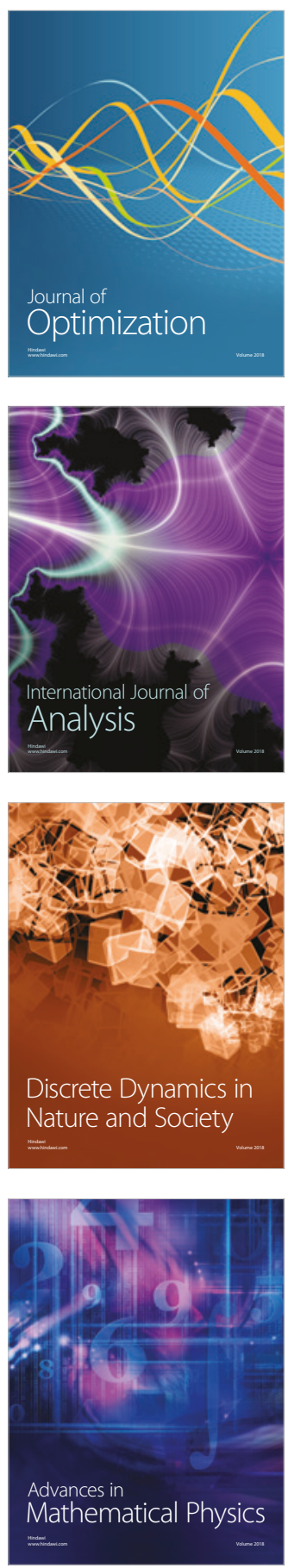